\title{
New Method for Image Analyze using Mathematical Method of Graph theory and it's Application in Robot Laser Hardening
}

\author{
Matej Babič * \\ Faculty of Education in Maribor
}

Matej Babic born 7th March 1983. He received his Ph.D. degree in Computer Science from the Faculty of Electrical Engineering and Computer Science. He studied Mathematics at the Faculty of Education in Maribor. Current position: Independend Ph.D researcher. He research interests are fractal geometry, graph theory, intelligent systems, hybrid machine learning, and the topography of materials after hardening.

\begin{abstract}
Graphs are structures that have a long history in mathematics and have been applied in almost every scientific and engineering field. Use of graphs has become very influential in computer science and has led to many applications in denoising, enhancement, restoration, and object extraction. Accounting for the wide variety of problems being solved with graphs in image processing and computer vision. We have two goals for this article. Firstly, we make this work selfcontained by reviewing the basic concepts and notations for visibility graphs in 3D space that are used process of robot laser hardening. In process of robot laser hardening we have many open problems. One of most difficul problem is how to analyze SEM images of microstructure of robot laser hardenend specimens and make prediction of topographycal properties with image properties. Second, we connect these concepts to image processing and analysis from a conceptual level and discuss implementation details. 3D visibility computations are central to any computer graphics application. Drawing graphs as nodes connected by links in $3 \mathrm{D}$ space is visually compelling but computationally difficult. In computational geometry and robot motion planning, a visibility graph is a graph of intervisible locations, typically for a set of points and obstacles in the Euclidean plane. Each node in the graph represents a point location, and each edge represents a visible connection between them. That is, if the line segment connecting two locations does not pass through any obstacle, an edge is drawn between them in the graph. Thus, we use method of construction of visibility graphs in 3D space in SEM images of microstructure of robot laser hardenend specimens. At the end we use method of intelligent systems to predict topographical property of robot laser hardened specimens. With intelligent system we increase production of process of laser hardening, because we decrease time of process and increase topographical property of materials.
\end{abstract}

Keywords: Visibility graphs, image processing, materials science, intelligent systems.

\section{Introduction}

Visibility graphs [1-3] in 3D space are support for graduate courses in image processing and computer vision, it is also perfect as a reference for practicing 
engineers working on development and implementation of SEM image processing and analysis algorithms. Visibility graphs may be used to find Euclidean shortest paths among a set of polygonal obstacles in the plane: the shortest path between two obstacles follows straight line segments except at the vertices of the obstacles, where it may turn, so the Euclidean shortest path is the shortest path in a visibility graph that has as its nodes the start and destination points and the vertices of the obstacles. Visibility calculations are central to any computer graphics application. In imaging science, image processing is any form of signal processing for which the input is an image, such as a photograph or video frame; the output of image processing may be either an image or a set of characteristics or parameters related to the image. Most image-processing [4] techniques involve treating the image as a two-dimensional signal and applying standard signal-processing techniques to it. Image processing is a method to convert an image into digital form and perform some operations on it, in order to get an enhanced image or to extract some useful information from it. It is a type of signal dispensation in which input is image, like video frame or photograph and output may be image or characteristics associated with that image. Usually Image Processing system includes treating images as two dimensional signals while applying already set signal processing methods to them. It is among rapidly growing technologies today, with its applications in various aspects of a business. Image Processing forms core research area within engineering and computer science disciplines too. Laser hardening [5] is a metal surface treatment process that is complementary to conventional aim and induction hardening processes. A high-power laser beam is used to heat a metal surface rapidly and selectively so as to produce hardened case depths of up to $1.5 \mathrm{~mm}$ with the hardness of a martensitic micro-structure, providing improved properties such as wear resistance and increased strength. In this work, we have used a scanning electronic microscope (SEM) to discover and analyse the topological property of visibility graph in 3D space of microstructure of robot laser hardened specimens. The present study is intended to use the new method of constructing visibility graphs in 3D space and its topological property to describe completely the mechanical properties hardness of robot laser hardened specimens. Finally, the concept of visibility graphs in $3 D$ is applied to characterize the microstructure and derive useful relationships between the topological properties density of 3D visibility graphs and microstructural features. Topological properties of hardened specimens was obvious in [6], but not with method visibility graphs, and not with intelligent method genetic programing. In this article, we also use new method to describe properties of hardened specimens with methods of intelligent systems [7]. Today technologists who operate various CNC machines only have knowledge based on practical experience. Each technologist must consider numerous CNC machine parameters to get the best desired results. Because this is a very time-consuming process, we used the method of intelligent systems, which allows us to obtain results more quickly. Also, the aim of the contribution is to outline possibilities of applying artificial multiple regression, neural networks and genetic programming for the prediction of mechanical steel properties after robot laser heat treatment and to judge their perspective use in this field.

\section{Experimental Data}

Digital image processing operates on sampled data from an underlying continuous light field Each sample is called a pixel in 2D or a voxel in 3D. The sampling is based on the notion of lattice, which can be viewed as a regular tiling of a space by a primitive cell. Firstly, we use program ImageJ to convert SEM images to 3D images of SEM images. Then, we find all point of 3D images od SEM images. We find coodinates $(x, y, z)$ of graphs of 3D images od SEM images. After this process, we use algorithm for constructing 3D visibility graphs. And in the end, we calculate topological property of 3D visibility graphs. We, calculated density of $3 \mathrm{D}$ visibility graphs. On fig. 1 is presented image processing of SEM image of robot laser hardened specimens.

Solution of visibility graph in 3D space is obvious, like algorithm for constructing visibility graphs in 3D space in [8]. Visibility graphs of a series of digitised surface microstructures from the surface of the robot laser modified specimens indicate that useful correlations can be derived between the topological properties of 3D visibility graphs 


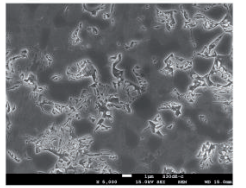

SEM IMAGE

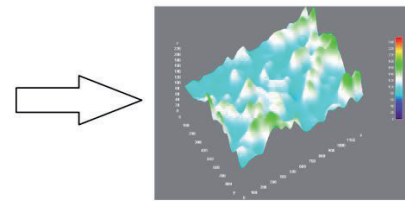

3D IMAGE OF SEM IMAGE

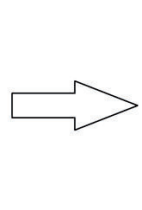

POINT OF 3D IMAGE OF SEM IMAGE
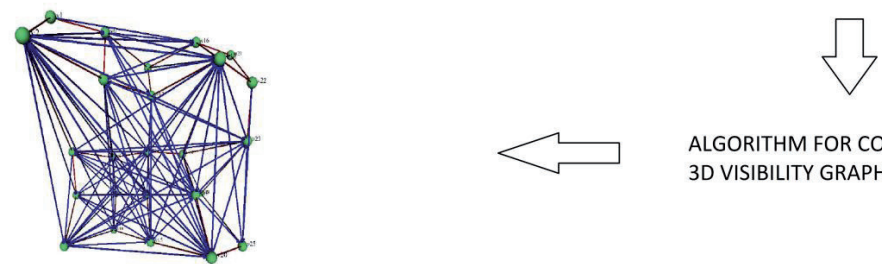

ALGORITHM FOR CONSTRUCTING 3D VISIBILITY GRAPHS

CALCULATE TOPOLOGICAL PROPERTY OF 3D VISIBILITY GRAPHS

Fig. 1: Image processing of SEM image of robot laser hardened specimens.

and the surface microstructure features, such as harness. In visibility graph in 3D sprace we calculated density of graph with program Pajek [9]. Pajek software and data sets are available so readers can learn network analysis through application and case studies.

To avoid unnecessarily high classification performance of our model, data were divided into training and testing data sets. Data of 22 specimens were randomized from the database and used for GP modelling. The theory of GP can be found in many books and articles that deal with evolutionary computation $[10,11]$. GP is probably the most general approach of evolutionary computational methods. In GP the structures subject to adaptation are the hierarchically organized computer programmes (organisms) the size and form of which dynamically change during simulated evolution. Genetic programming is a collection of methods for the automatic generation of computer programs that solve carefully specified problems, via the core, but highly abstracted principles of natural selection. In a sentence, it is the compounded breeding of (initially random) computer programs, where only the relatively more successful individuals pass on genetic material (programs and program fragments) to the next generation. The following evolutionary parameters were selected for the process of simulated evolutions: 500 for the size of the population of organisms, 100 for the maximum number of generations, 0.4 for the reproduction probability, 0.6 for the crossover probability, 6 for the maximum permissible depth in the creation of the population, 10 for the maximum permissible depth after the operation of crossover of two organisms, and 2 for the smallest permissible depth of organisms in generating new organisms. Genetic operations of reproduction and crossover were used. For selection of organisms the tournament method with tournament size 7 was used. In Fig. 2 is presented tree crossover in genetic programming. In Fig. 3 is presented model of genetic programming.
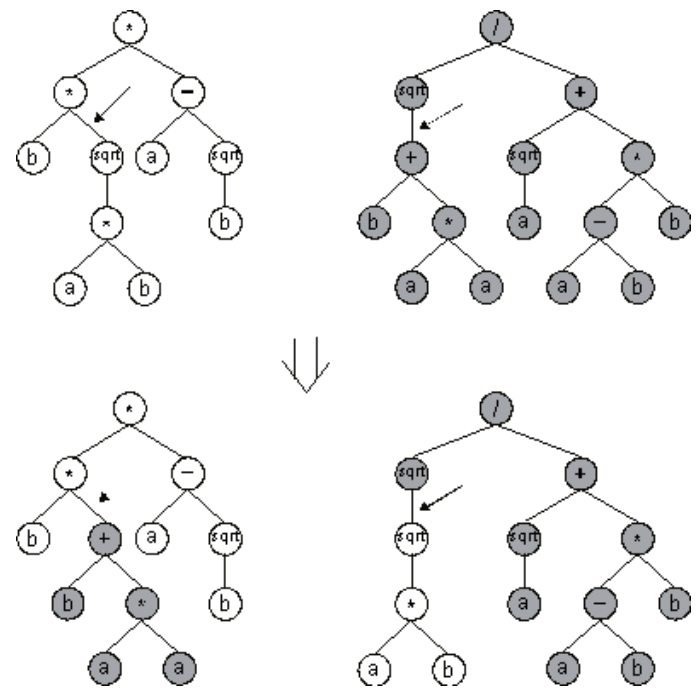

$\checkmark$

Fig. 2: Tree crossover in genetic programming. The arrows indicate the crossover points. 


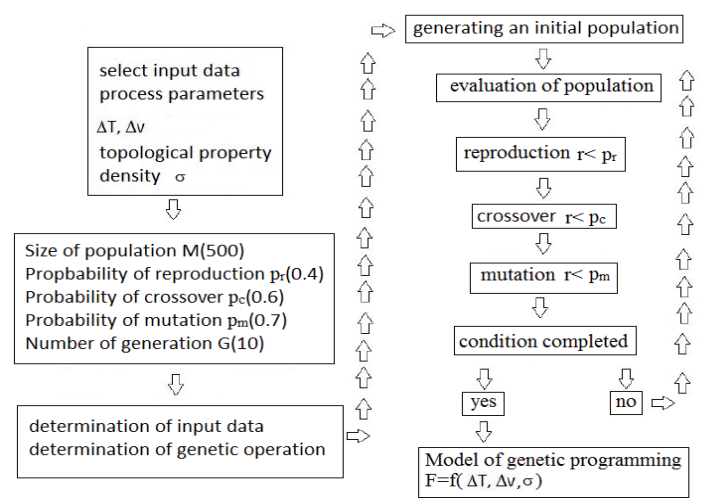

Fig. 3: Model of genetic programming.

A series of algorithms that attempt to identify underlying relationships in a set of data by using a process that mimics the way the human brain operates. Neural networks [12] have the ability to adapt to changing input so that the network produces the best possible result without the need to redesign the output criteria. Rather than using a digital model, in which all computations manipulate zeros and ones, a neural network works by creating connections between processing elements, the computer equivalent of neurons. The organization and weights of the connections determine the output. Neural networks are particularly effective for predicting events when the networks have a large database of prior examples to draw on. Strictly speaking, a neural network implies a nondigital computer, but neural networks can be simulated on digital computers. Neural networks can have any number of layers, and any number of nodes per layer. Most applications use the three layer structure with a maximum of a few hundred input nodes. The hidden layer is usually about 10\% the size of the input layer. In the case of target detection, the output layer only needs a single node. The output of this node is thresholded to provide a positive or negative indication of the target's presence or absence in the input data. Fig. 4 present The general multi-layer neural network system. Fig. 5 present model of neural network.

Many multiple regression [13] models contain variables whose $t$ statistics have nonsignificant $P$ values. These variables are judged to have not displayed statistically significant predictive capability in the presence of the other predictors.

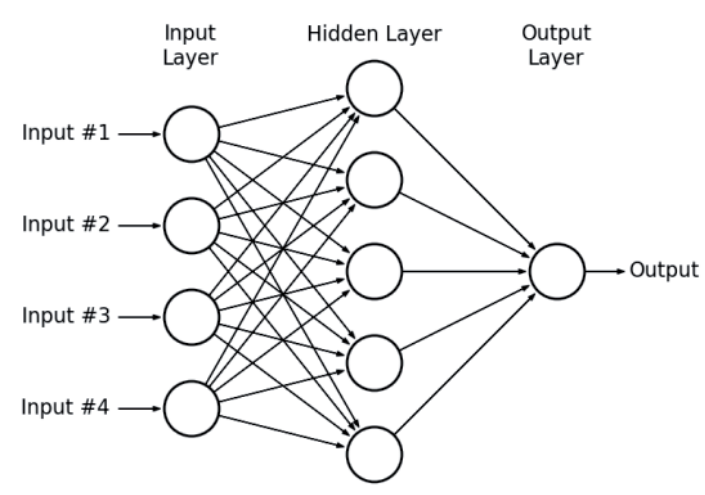

Fig. 4: The general multi-layer neural network system.

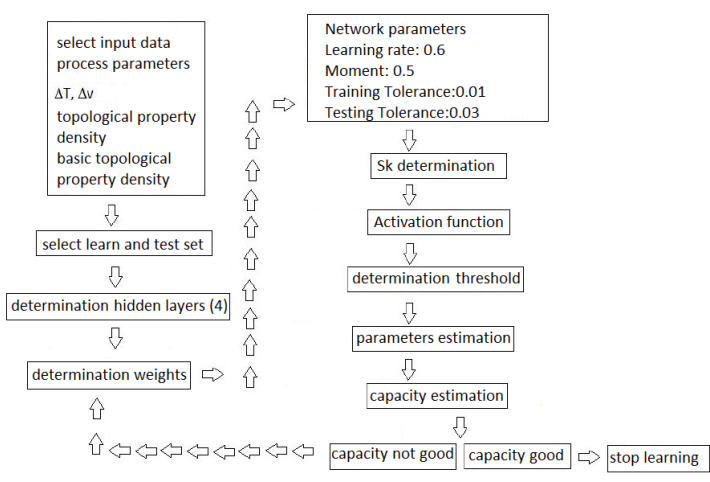

Fig. 5: Model of neural network.

The question is then whether some variables can be removed from the model. To answer this question, many models are examined to find the one that's best in some sense. In multiple regression, more than one variable is used to predict the criterion. Predicted scores from multiple regression are linear combinations of the predictor variables. Therefore, the general form of a prediction equation from multiple regression is presented bellow.

Model of multiple regression

\section{$\mathrm{Y}=\mathrm{b}+\mathrm{b} 1 \mathrm{X} 1+\mathrm{b} 2 \mathrm{X} 2+\ldots+\mathrm{bmXm}+\varepsilon$}

Fig. 6 present intelligent system for modelling data. Firstly, we use input experimental data. This data are density of visibility graphs in 3D space, temperature and speed of robot laser cell and basic hardness of hardened specimens. Then we use method of intelligent system, genetic programming, neural network and multiple regression to predict topographical property hardness of hardened specimens. 


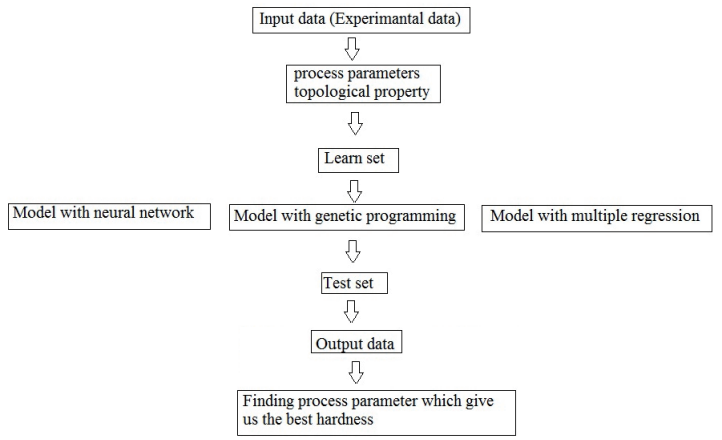

Fig. 6: Intelligent system for modelling data.

\section{Results and Discussion}

In Table 1, the parameters of hardened specimens that impact on roughness are presented. We mark specimens from P1 to P22. Parameter X1 presents the parameter of temperature in degree of Celsius $[C], X 2$ presents the speed of hardening $[\mathrm{mm} / \mathrm{s}]$ and $X 3$ presents density of visibility graphs in $3 D$ space. The last parameter $Y$ is the measured surface hardness of laser-hardened robot specimens. Table 2 presents experimental and prediction data regarding the surface hardness of laser hardened robot specimens. In Table 2 present symbol $\mathrm{S}$ name of specimens, E experimental data, NM1 prediction with neural network with 36\% learn set, NM2 prediction with neural network with $50 \%$ learn set, NM3 prediction with neural network with method one live out, $R$ prediction with regression and GP prediction with genetic programming. In Table 1, we can see that specimen P20 has the largest density of visibility graphs in 3D; 0.2960 , thus specimen P20 is most complex. Specimen P1 is has most hardness after hardening, that is $60 \mathrm{HRC}$. The measured and predicted surface roughness of robot laser hardened specimens is shown in the graph in Fig. 8. The genetic programming model is presented on Fig. 7. The genetic programming model presents a 1,33\% deviation from the measured data, which is less than the regression model, which presents a 4,47\% deviation. The best neural network present 1,42\% deviation from the measured data.

Table 1: Parameters of hardened specimens.

\begin{tabular}{|l|l|l|l|l|}
\hline S & X1 & X2 & X3 & Y \\
\hline P1 & 1000 & 2 & 0,1936 & 60 \\
\hline P2 & 1000 & 3 & 0,2208 & 58,7 \\
\hline P3 & 1000 & 4 & 0,2144 & 56 \\
\hline P4 & 1000 & 5 & 0,2256 & 56,5 \\
\hline P5 & 1400 & 2 & 0,2445 & 58 \\
\hline P6 & 1400 & 3 & 0,2221 & 57,8 \\
\hline P7 & 1400 & 4 & 0,2036 & 58,1 \\
\hline P8 & 1400 & 5 & 0,2096 & 58,2 \\
\hline P9 & 1000 & 2 & 0,2352 & 57,4 \\
\hline P10 & 1000 & 3 & 0,2288 & 56,1 \\
\hline P11 & 1000 & 4 & 0,2144 & 53,8 \\
\hline P12 & 1000 & 5 & 0,2352 & 56 \\
\hline P13 & 1400 & 2 & 0,2208 & 55,3 \\
\hline P14 & 1400 & 3 & 0,232 & 57,2 \\
\hline P15 & 1400 & 4 & 0,1984 & 57,8 \\
\hline P16 & 1400 & 5 & 0,1904 & 58 \\
\hline P17 & 800 & 0 & 0,2832 & 52 \\
\hline P18 & 1400 & 0 & 0,2688 & 57 \\
\hline P19 & 2000 & 0 & 0,2416 & 56 \\
\hline P20 & 950 & 0 & 0,2128 & 58 \\
\hline P21 & 850 & 0 & 0,208 & 57 \\
\hline P22 & 0 & 0 & 0,296 & 34 \\
\hline & & & & \\
\hline
\end{tabular}

Fig. 7: Model of genetic programming.

\section{Model of regression}

$$
Y=71,82942368+0,006682914 \times X 1-0,172405205 \times X 2-102,024491 \times X 3
$$


Table 1: Experimental and prediction data.

\begin{tabular}{|l|l|l|l|l|l|l|}
\hline S & E & NM1 & NM2 & NM3 & R & GP \\
\hline P1 & 60 & 60,19329 & 57,966766 & 57,756421 & 58,415585 & 60,0202 \\
\hline P2 & 58,7 & 57,98771 & 58,116888 & 57,757294 & 55,468114 & 57,2989 \\
\hline P3 & 56 & 56,83474 & 56,343353 & 56,094604 & 55,948666 & 54,9596 \\
\hline P4 & 56,5 & 56,26917 & 56,091986 & 56,008197 & 54,633586 & 57,534 \\
\hline P5 & 58 & 58,16926 & 58,133472 & 57,008087 & 55,895704 & 57,8244 \\
\hline P6 & 57,8 & 57,86378 & 58,152673 & 57,763403 & 58,008648 & 57,2845 \\
\hline P7 & 58,1 & 57,96153 & 57,889087 & 57,752057 & 59,723695 & 57,1712 \\
\hline P8 & 58,2 & 57,99731 & 56,305823 & 57,062042 & 58,939143 & 57,6666 \\
\hline P9 & 57,4 & 59,58145 & 57,069525 & 57,763403 & 54,171367 & 58,1123 \\
\hline P10 & 56,1 & 57,79744 & 58,136963 & 57,761658 & 54,651918 & 57,2081 \\
\hline P11 & 53,8 & 56,83474 & 56,343353 & 56,094604 & 55,948666 & 54,9596 \\
\hline P12 & 56 & 56,10857 & 56,227271 & 56,044855 & 53,654151 & 57,4552 \\
\hline P13 & 55,3 & 58,61526 & 58,148309 & 57,761658 & 58,313685 & 55,0581 \\
\hline P14 & 57,2 & 57,70929 & 58,152673 & 58,776495 & 56,998605 & 57,1703 \\
\hline P15 & 57,8 & 58,02786 & 57,561786 & 57,738965 & 60,254223 & 57,3491 \\
\hline P16 & 58 & 58,1937 & 56,092859 & 56,011688 & 60,898013 & 57,8311 \\
\hline P17 & 52 & 60,78505 & 56,09024 & 51,981085 & 48,282419 & 50,6778 \\
\hline P18 & 57 & 60,38007 & 56,09024 & 56,964789 & 53,761319 & 54,6548 \\
\hline P19 & 56 & 59,49243 & 57,991205 & 56,327643 & 60,546134 & 56,8075 \\
\hline P20 & 58 & 60,85923 & 56,09024 & 57,773 & 60,54613369 & 56,8075 \\
\hline P21 & 57 & 60,8636 & 56,09024 & 56,9613 & 56,46737984 & 57,4179 \\
\hline P22 & 34 & 60,77108 & 42,09024 & 34,29611 & 56,28880604 & 57,464 \\
\hline
\end{tabular}

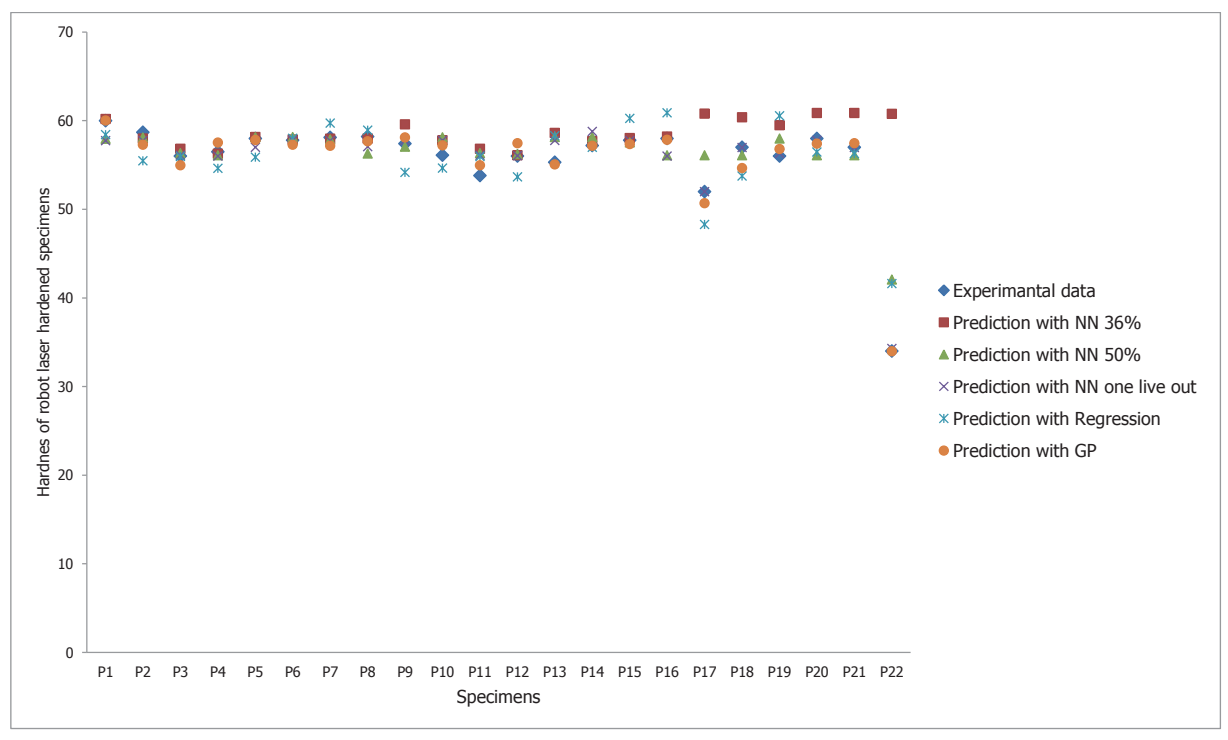

Fig. 8: The measured and predicted surface hardness of robot laser hardened specimens. 


\section{Discussion}

The visibility graph problem itself has long been studied in computational geometry and has been applied to a variety of areas. We use method visibility graph in image processing. All SEM images of robot laser hardened specimens, we analyze with topological property density of visibility graphs. The surface of the hardened material exhibits a more complex network than at depth, which is to be expected, because the laser beam is focused directly on the surface. Thus, it was necessary to develop intelligent systems based on individual samples to disclose the topological properties of a material after heat treatment. Intelligent system give us good results. We use three intelligent system methods; genetic programming, neural network and multiple regression to predict the hardness of laser hardened robot specimens. We show that the genetic programming model gives us the best predicted results. The neural network model is better than the regression model and as good as the genetic programming model.

\section{Conclusions}

The paper presents new method for image analyze using mathematical method of graph theory and it's application in Materials Science. We present method of visibility graph in 3D space to analyze complexity of robot laser hardened specimens. Analysing complexity of robot laser hardened surfaces is very hard problem. New presented method have many applications in pattern recognition, computer graphics, computational geometry etc. The main findings can be summarised as follows:

$\checkmark$ We use method visibility graph in 3D space and it's topological property to image procesing on SEM images of robot laser hardened specimens.

$\checkmark$ We describe the topographic properties of the hardened specimens by using the topological properties of the visibility graphs in $3 D$ space.

$\checkmark$ We describe the relationship between hardness and the parameters of the robot laser cell by using the topological properties of the $3 D$ visibility graphs. This finding is important with regard to certain alloys that are hard to mix because they have different melting temperatures; however, such alloys have better technical characteristics. By varying different parameters (e.g., temperature), robot laser cells produce different patterns with different topological properties of $3 D$ visibility graphs. $\checkmark$ For prediction of hardness of hardened specimens we use neural network, genetic algorithm and multiple regression.

$\checkmark$ With intelligent system we increase production of process of laser hardening, because we decrease time of process and increase topographical property of materials.

\section{References}

[1] Ben-Moshe, O. Hall-Holt, M.J. Katz and J.S.B. Mitchell. (2004). Computing the Visibility Graph of Points within a Polygon, Proc. 20th ACM Symp. on Computational Geometry, 27-35.

[2] Berg, Mark; van Kreveld, Marc; Overmars, Mark; Schwarzkopf, Otfried (2000), "Chapter 15: Visibility Graphs,' Computational Geometry (2nd ed.), Springer-Verlag, pp. 307-317, ISBN 3-540-65620-0.

[3] Lozano-Pérez, Tomás; Wesley, Michael A. (1979), An algorithm for planning collision-free paths among polyhedral obstacles, Communications of the ACM 22 (10): 560-570, doi:10.1145/359156.359164

[4] A. Chambolle, R. A. DeVore, N.-Y. Lee and Î. J. Lucier "Nonlinear wavelet image processing: variational problems, compression, and noise removal through wavelet shrinkage", IEEE Trans. Image Processings, vol. 7, no. 33, pp.319 $-3351998$

[5] M. Babič, M. Milfelner, S. Stepišnik, Laser hardening metals. In: Perme, T., Švetak, D., Balič, J. (eds.), IRT Industrial Forum, Portoroz, 7-8 June 2010. Source of knowledge and experience for the profession: Proceedings of the Forum. Skofljica: Profidtp.

[6] Babič, Matej, Kokol, Peter, Belič, Igor, Panjan, Peter, Kovačič, Miha, Balič, Jože, Verbovšek, Timotej. Prediction of the hardness of hardened specimens with a neural network = Napoved trdote kaljenih vzorcev z nevronskimi mrežami. Materiali in tehnologije, ISSN 1580-2949. [2014, let. 48, št. 3, str. 409-414, ilustr. http://mit.imt.si/Revija/

[7] Hutter, M. (2012). "One Decade of Universal Artificial Intelligence". Theoretical Foundations of Artificial General Intelligence. Atlantis Thinking Machines 4. doi:10.2991/978-9491216-62-6_5. ISBN 978-94-91216-61-9.

[8] Matej Babič. Doctoral dissertation. 2014

[9] Nooy, Wouter de, Andrej Mrvar, Vladimir Batagelj. (2005). ExploratorySocial Network Analysis with Pajek. New York: CambridgeUniversity Press.

[10] Koza, John (1992). Genetic Programming: On the Programming of Computers by Means of Natural Selection. Cambridge, MA: MIT Press. ISBN 978-0262111706.

[11] Bäck, T.; Hammel, U.; Schwefel, H.-P. Evolutionary computation: Comments on the history and current state. IEEE 
Acta Mechanica Slovaca

Journal published by Faculty of Mechanical Engineering - Technical University of Košice

Transaction on Evolutionary Computation 1 (1997) 1, 3-17

[12] Graves, Alex; and Schmidhuber, Jürgen; Offline Handwriting Recognition with Multidimensional Recurrent Neural Networks, in Bengio, Yoshua; Schuurmans, Dale; Lafferty, John; Williams, Chris K. l.; and Culotta, Aron (eds.), Advances in Neural Information Processing Systems 22 (NIPS'22), December 7th-10th, 2009, Vancouver, BC, Neural Information Processing Systems (NIPS) Foundation, 2009, pp. 545-552.

[13] Box, G. E. P. (1954). "Some Theorems on Quadratic Forms Applied in the Study of Analysis of Variance Problems, I. Effect of Inequality of Variance in the One-Way Classification". The Annals of Mathematical Statistics 25 (2): 290. doi:10.1214/aoms/1177728786. 